\title{
Automated Correlative Tomography of an Aluminum 7075 Alloy Spanning Length Scales and Modalities
}

\author{
Arno P. Merkle ${ }^{1}$, Lorenz Lechner ${ }^{1}$, Luke Hunter ${ }^{1}$, Jeff Gelb ${ }^{1}$, S. S. Singh ${ }^{2}$, N. Chawla ${ }^{2}$ \\ ${ }^{1}$ Carl Zeiss X-ray Microscopy, Inc. 4385 Hopyard Road, Pleasanton, CA, USA \\ ${ }^{2}$ Arizona State University, 1151 S. Forest Ave., Tempe, AZ, USA
}

The recent confluence of advanced computing power with new 3D characterization approaches has yielded great enthusiasm in the materials science community to pursue new scientific pathways, supported by the 'materials by design' approach. It has become clear that one technique by itself cannot span all of the necessary length-scales in materials characterization nor provide a complete set of modalities. There is need in several areas such as energy materials, electronics, and metals, where the combination of two or more techniques is required to obtain a fundamental understanding of the microstructure [1].

Here we detail a study on light-weight structural alloys, which has benefitted from characterization of the same sample volume across multiple length scales[2]. A new, efficient 3D correlative microscopy workflow is presented, that utilizes a non-destructive submicrometer tomographic imaging approach[3, 4], 3D X-ray microscopy (XRM), to guide focused ion beam and scanning electron microscopy (FIB-SEM), in order to reveal targeted sub-surface regions of interest at high resolution. This approach, enabled by the emergence of an integrated, modern workflow environment (ZEISS Atlas 5), points to the future of efficient correlation in 3D across modalities and length scales.

An aluminum 7075 alloy was investigated, exhibiting hierarchical structures that required characterization in 3D across multiple length scales, modalities and instruments. Al 7075 alloys are used extensively in structural applications due to their high strength-to-weight ratio. The microstructure of the alloy contains precipitates, constituent particles (also called inclusions), and pores associated with the inclusions. The precipitates are intentionally formed through heat treatment to provide strength to the alloy, whereas constituent particles are undesirable and form during alloy casting. The size, shape, and distribution of these microstructural features in the alloy are known to affect the corrosion and mechanical behavior (fatigue, tensile, and stress corrosion cracking) [5]. Therefore, it is necessary to obtain combined information of the size, shape, and distribution of the precipitates, constituent particles, and pores, in three dimensions (3D) and from the same volume of interest. Here we present the experimental methodology of combining tomographic length scales, as well as the approach to extracting key $3 \mathrm{D}$ microstructural quantification.

By utilizing XRM to automate FIB-SEM tomography acquisition at specific locations via Atlas 5, we demonstrate the ability to efficiently seek and acquire volumes of interest, greatly increasing the utilization potential of the FIB-SEM. 
References:

[1] Burnett T., et. al. Scientific Reports, (2014)

[2] A. P. Merkle et al., Microscopy and Analysis, 28 (2014), p. S10-S13

[3] A. P. Merkle and J. Gelb, Microscopy Today, 21 (2013), p. 10

[4] E Maire and P Withers, International Materials Review, 59 (2014), p. 1

[5] Singh, S. et al., Mater. Res. Lett. 2 (2014), p. 217

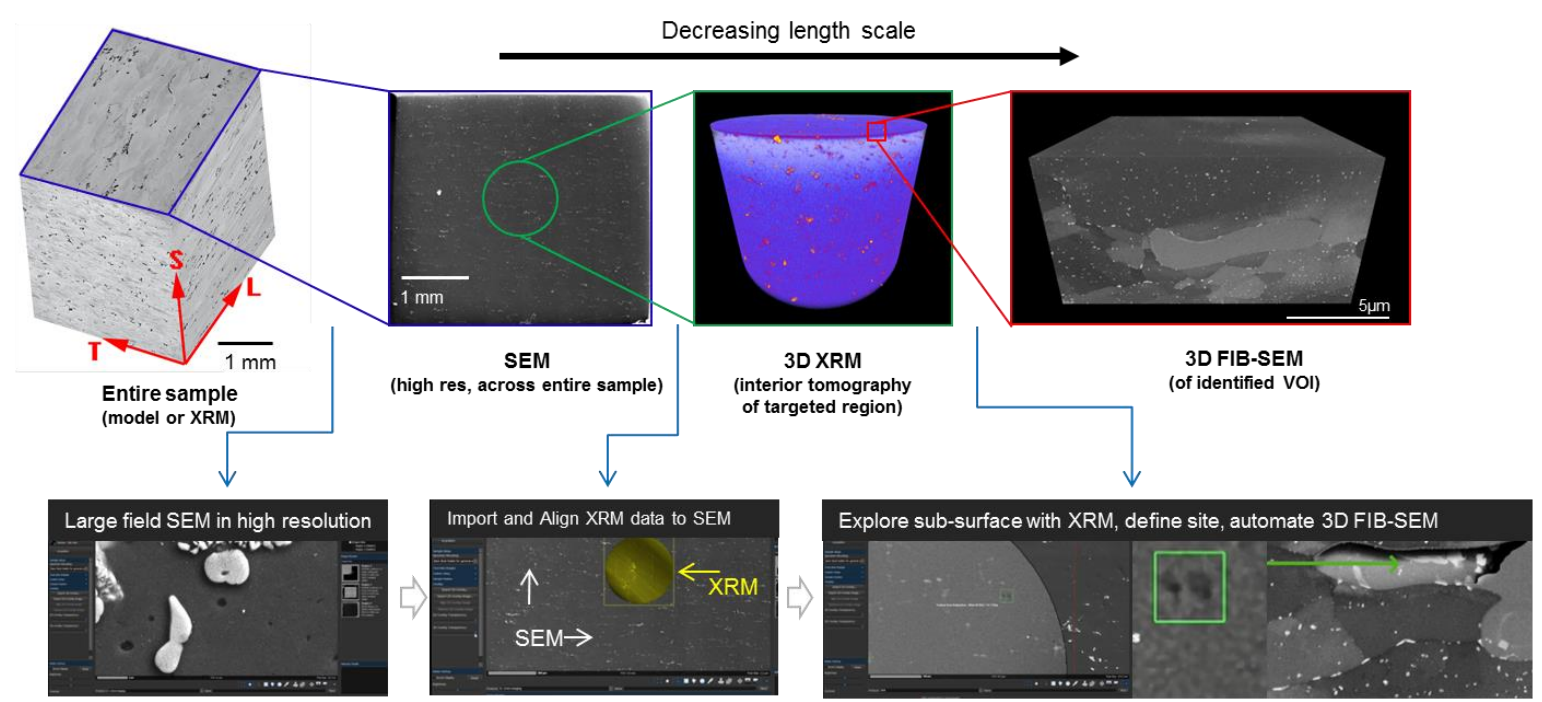

Figure 1: A 3D automated correlative workflow demonstrated on an Aluminum 7075 alloy to access information about inclusions, voids, precipitates and the Al matrix grain structure.

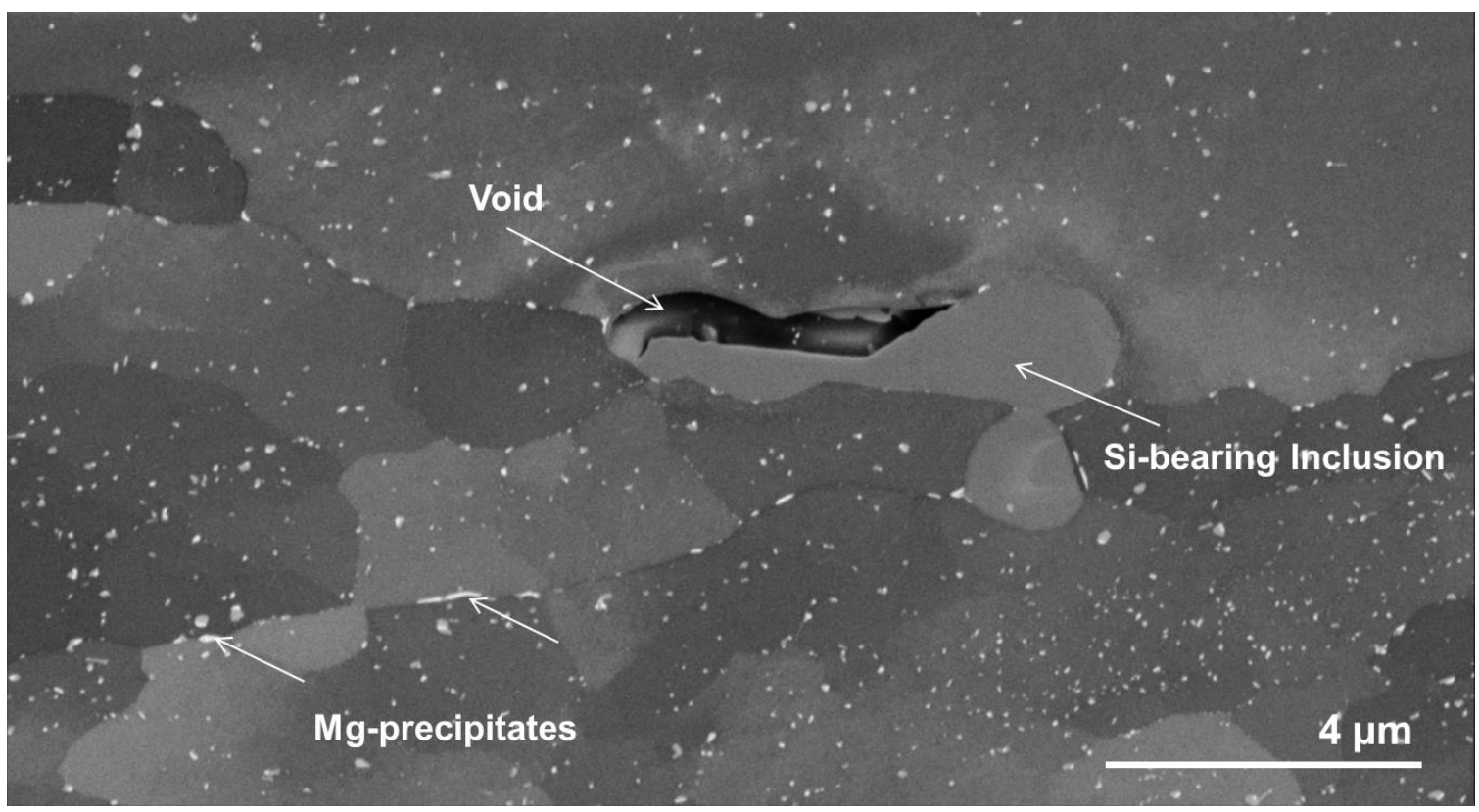

Figure 2 - 2D SEM image from the 3D FIB-SEM tomography acquisition, confirming the location of both a Si-bearing inclusion and void were identified, in the center of the region of interest. Multiple such sites may be programmed for automated FIB-SEM acquisition. 